\title{
Letter to Editor: Role of Industry in the Fight Against COVID-19 Crisis
}

\author{
Pooya Parvizi ${ }^{1}$, Milad Jalilian ${ }^{2 \star}$ (D), Hana Parvizi ${ }^{3}$, Sara Amiri $^{4}$
}

1. School of Engineering, University of Birmingham, Birmingham, United Kingdom

2. Department of Physics, Faculty of Science, Razi University, Kermanshah, Iran.

3. Department of Science, Faculty of Science, University of British Columbia, Vancouver, Canada.

4. School of Medicine, Kermanshah University of Medical Sciences, Kermanshah, Iran.

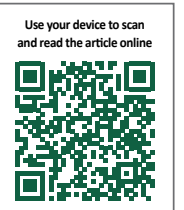

ditation: Parvizi P, Jalilian M, Parvizi H, Amiri S. Role of Industry in the Fight Against COVID-19 Crisis. Health in Emergencies and Disasters Quarterly. 2021; 6(3):129-132. http://dx.doi.org/10.32598/hdq.6.3.387.1

http://dx.doi.org/10.32598/hdq.6.3.387.1

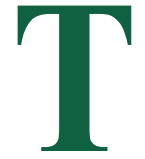

he first case of COVID-19 was reported in December 2019 in Wuhan, China. Until December 1, 2020, the number of confirmed cases and related deaths are 61.8 million and 1.4 million, respectively $[1,2]$. After the incubation period, between 2 to 14 days, common symptoms such as fever, cough, respiratory problems, and fatigue are seen in the infected person [3-5]. In addition to human health, the pandemic threatens countries' economies and has adverse effects on the global economy $[6,7]$, and the production sector, as the mainstay of the economy, is not an exception. Activating the production industries (chemical manufacturing, medical devices manufacturing, food processing, metal manufacturing, plastics industry, and pharmaceutical manufacturing) as the main branch of the economy and meeting the needs of the people and society can significantly reduce the adverse effects of this pandemic.

The manufacturing industry's fight against COVID-19 may include but is not limited to producing medical equipment (raw materials of equipment, masks, face shields, etc.), meeting the basic needs of the people (food and sanitation facilities), preventing the unemployment rate from rising, and reducing economic pressures in countries. (countries with better economic conditions allocate more financial resources to fight the crisis.)
In this regard, maintaining the physical and mental health of employees is of great importance (the presupposition of any measure adopted should be to protect the health of employees) [8]. The formation of crisis committees in companies is essential to implement and monitor the health guidelines to maintain employees' health. Some recommended control measures are derived from a variety of sources and ideas [9-11].

The steps that employers in the manufacturing industry must take are as follow:

- Implementation of social distancing protocols and restricts gatherings,

- Encourage employees with symptoms to stay at home,

- Disinfect sensitive points (staff entry and exit points, shoe soles, surfaces, and objects),

- Holding virtual business meetings (video conferencing, teleconferencing, social media, and email),

- Frequent control of the body temperature of employees,

- Perform diagnostic tests at specific time intervals (priority with suspicious people with the underlying disease),

- Employees screening to identify cases with suspected disease and or underlying diseases, 
- Raising employees' awareness in recognizing common COVID-19 symptoms and ways to prevent them by installing posters, posting statements in integrated management systems, using audio systems, and industrial paging (using credible information sources),

- Eliminate public transportation for staff relocation,

- Disinfect high-risk and common points between shifts,

- Placing antiseptic dispensers in different parts of the company,

- Allocate specified containers to collect used masks, gloves, and gowns,

- Manage working hours and staff telecommuting,

- Psychological counseling to help mental health and control stress in employees,

- Providing immune-modulatory supplements such as Vitamin C, Vitamin D, Zinc, Selenium and virustatic drugs such as Ivermectin to strengthen the immune system of the employees and prevent the transmission of the disease.(Note: Consult medical doctor before use to avoid drug interactions and medication complications),

- Ultraviolet light can be used to disinfect high-risk areas such as public toilets, dining halls, and high traffic areas (Note: to prevent UV side effects, UV light safety goggles must be used and this operation can be done after staff's working hours), and

- Creating suitable environmental conditions for employees (in terms of temperature and ventilation systems).

The actions that the employees must take include:

- Frequent hand washing with warm soapy water for 20 seconds,

- Using face masks, gloves, and face shields,

- Prevent stressful rumors about coronavirus in the workplace,

- Do not gather in the workplace,

- Practice social distance, and

- Avoid going to work when the first symptoms appear.
After the end of the COVID-19 pandemic, the world enters into the post-coronavirus phase, and the assessment of the effects left by it begins. Specialized studies of the economic effects of this crisis on various industries, the unemployment rate of industrial workers, employees health assessment (physically and mentally), and the prevalence (infection and death) among industrial workers can be of great help in developing preventive and control guidelines in facing future pandemic diseases. In other words, it is crucial to draw accurate conclusions about the effects of this crisis and to learn from it to be more prepared to fight future crises.

\section{Ethical Considerations}

\section{Compliance with ethical guidelines}

All ethical principles are considered in this article.

\section{Funding}

The authors received no financial support for the research, authorship, and or publication of this article.

\section{Authors' contributions}

All authors equally contributed to preparing this article.

\section{Conflict of interest}

The authors declared no conflict of interest.

\section{References}

[1] Zhu N, Zhang D, Wang W, Li X, Yang B, Song J, et al. A novel coronavirus from patients with pneumonia in China, 2019. The New England Journal of Medicine. 2020; 382(8):727-33. [DOI:10.3390/ jcm9020538] [PMID] [PMCID]

[2] World Health Organization. Coronavirus Disease (COVID-2019) situation report [Internet]. 2020 [Updated 2020 January 21]. Available from: https://wwwwhoint/emergencies/diseases/novelcoronavirus-2019/situation-reports/

[3] Linton NM, Kobayashi T, Yang Y, Hayashi K, Akhmetzhanov $\mathrm{AR}$, Jung SM, et al. Incubation period and other epidemiological characteristics of 2019 novel Coronavirus infections with right truncation: A statistical analysis of publicly available case data. Journal of Clinical Medicine. 2020; 9(2):538. [DOI:10.3390/ jcm9020538] [PMID] [PMCID]

[4] Rothan HA, Byrareddy SN. The epidemiology and pathogenesis of Coronavirus Disease (COVID-19) outbreak. Journal of Autoimmunity. 2020; 109:102433. [DOI:10.1016/j.jaut.2020.102433] [PMID] [PMCID]

[5] Parvizi P, Jalilian M, Parvizi H, Amiri S, Mohammad Doust H. The COVID-19 pandemic: Data analysis, impacts and future considerations. Iranian Journal of Medical Microbiology. 2021; 15(1):1-17. [DOI:10.30699/ijmm.15.1.1] 
[6] Fineberg HV. Ten weeks to crush the curve. The New England Journal of Medicine. 2020; 382(17):e37. [DOI:10.1056/ NEJMe2007263] [PMID]

[7] Madhav N, Oppenheim B, Gallivan M, Mulembakani P, Rubin E, Wolfe N. Pandemics: Risks, impacts, and mitigation. In: Jamison DT, Gelband H, Horton S, Jha P, Laxminarayan R, Mock CN, et al., editors. Disease control priorities: Improving health and reducing poverty. Washington (DC): The International Bank for Reconstruction and Development / The World Bank; 2017. [DOI:10.1596/978-1-4648-0527-1_ch17] [PMID]

[8] Biallas B, Froböse I, Zöller M, Wilke C. [Analysis of workplace health promotion and its effect on work ability and health-related quality of life in a medium-sized business (German)]. Gesundheitswesen (Bundesverband der Arzte des Offentlichen Gesundheitsdienstes (Germany). 2015; 77(5):357-61. [PMID] [DOI:10.1596/978-1-4648-0527-1_ch17]

[9] Centers for Disease Control and Prevention. Interim guidance for businesses and employers to plan and respond to coronavirus disease 2019 (COVID-19) [Internet]. 2020 [Updated February 26]. Availble from: https://stacks.cdc.gov/ view/cdc/85488

[10] World Health Organization. Getting your workplace ready for COVID-19: How COVID-19 spreads [Internet]. 2020 [Updated 2020 March 19]. Availble from: https://apps.who.int/ iris/handle/10665/331584

[11] White W. Business guide to COVID-19: Prevention and mitigation strategies for manufacturing [Internet]. 2020 [Updated 2020]. Available from: https://gamep.org/wp-content/uploads/2020/03/Business-Guide-for-COVID-19.pdf 
This Page Intentionally Left Blank 